\title{
Deuterated methanol on a solar system scale around the HH212 protostar
}

\author{
E. Bianchi ${ }^{1,2}$, C. Codella ${ }^{1}$, C. Ceccarelli ${ }^{3,4,1}$, V. Taquet ${ }^{1}$, S. Cabrit ${ }^{5}$, F. Bacciotti ${ }^{1}$, R. Bachiller ${ }^{6}$, E. Chapillon ${ }^{7,8}$, \\ F. Gueth ${ }^{8}$, A. Gusdorf ${ }^{9}$, B. Lefloch ${ }^{3,4}$, S. Leurini ${ }^{10}$, L. Podio ${ }^{1}$, K. L. J. Rygl ${ }^{11}$, B. Tabone ${ }^{5}$, and M. Tafalla ${ }^{6}$ \\ ${ }^{1}$ INAF, Osservatorio Astrofisico di Arcetri, Largo E. Fermi 5, 50125 Firenze, Italy \\ e-mail: ebianchi@arcetri.astro.it \\ 2 Università degli Studi di Firenze, Dipartimento di Fisica e Astronomia, via G. Sansone 1, 50019 Sesto Fiorentino, Italy \\ 3 Univ. Grenoble Alpes, Institut de Planétologie et d'Astrophysique de Grenoble (IPAG), 38401 Grenoble, France \\ ${ }^{4}$ CNRS, Institut de Planétologie et d'Astrophysique de Grenoble (IPAG), 38401 Grenoble, France \\ 5 LERMA, Observatoire de Paris, PSL Research University, CNRS, Sorbonne Universités, UPMC Univ. Paris 06, \\ École Normale Supérieure, 75014 Paris, France \\ 6 IGN, Observatorio Astronómico Nacional, Alfonso XII 3, 28014 Madrid, Spain \\ 7 Laboratoire d'astrophysique de Bordeaux, Univ. Bordeaux, CNRS, B18N, allée Geoffroy Saint-Hilaire, 33615 Pessac, France \\ 8 IRAM, 300 rue de la Piscine, 38406 Saint-Martin-d'Hères, France \\ 9 LERMA, Observatoire de Paris, PSL Research University, CNRS, Sorbonne Universités, UPMC Univ. Paris 06, École Normale \\ Supérieure, 75005 Paris, France \\ 10 INAF-Osservatorio Astronomico di Cagliari, via della Scienza 5, 09047 Selargius (CA), Italy \\ 11 INAF-Istituto di Radioastronomia \& Italian ALMA Regional Centre, via P. Gobetti 101, 40129 Bologna, Italy
}

Received 19 June 2017 / Accepted 14 September 2017

\begin{abstract}
Context. Deuterium fractionation is a valuable tool for understanding the chemical evolution during the process that leads to the formation of a Sun-like planetary system.

Aims. Methanol is thought to be mainly formed during the prestellar phase, and its deuterated form keeps a memory of the conditions at that epoch. The unique combination of high angular resolution and sensitivity provided by ALMA enables us to measure methanol deuteration in the planet formation region around a Class 0 protostar and to understand its origin.

Methods. We mapped both the ${ }^{13} \mathrm{CH}_{3} \mathrm{OH}$ and $\mathrm{CH}_{2} \mathrm{DOH}$ distribution in the inner regions ( $\left.100 \mathrm{au}\right)$ of the $\mathrm{HH} 212$ system in Orion $\mathrm{B}$. To this end, we used ALMA Cycle 1 and Cycle 4 observations in Band 7 with angular resolution down to $\sim 0$ !' 15 .

Results. We detected 6 lines of ${ }^{13} \mathrm{CH}_{3} \mathrm{OH}$ and 13 lines of $\mathrm{CH}_{2} \mathrm{DOH}$ with upper level energies of up to $438 \mathrm{~K}$ in temperature units. We derived a rotational temperature of $(171 \pm 52) \mathrm{K}$ and column densities of $7 \times 10^{16} \mathrm{~cm}^{-2}\left({ }^{13} \mathrm{CH}_{3} \mathrm{OH}\right)$ and $1 \times 10^{17} \mathrm{~cm}^{-2}\left(\mathrm{CH}_{2} \mathrm{DOH}\right)$, respectively. This yields a $\mathrm{D} / \mathrm{H}$ ratio of $(2.4 \pm 0.4) \times 10^{-2}$, which is lower by an order of magnitude than previously measured values using single-dish telescopes toward protostars located in Perseus. Our findings are consistent with the higher dust temperatures in Orion B with respect to the temperature derived for the Perseus cloud. The emission traces a rotating structure extending up to 45 au from the jet axis, which is elongated by 90 au along the jet axis. So far, the origin of the observed emission appears to be related with the accretion disc. Only higher spatial resolution measurements will be able to distinguish between different possible scenarios, however: disc wind, disc atmosphere, or accretion shocks.
\end{abstract}

Key words. stars: formation - ISM: molecules - ISM: individual objects: HH212 - ISM: abundances

\section{Introduction}

Molecular deuteration is a powerful diagnostic tool for studying the past history of the gas associated with the formation of a proto-Sun and its protoplanetary system (see e.g. Ceccarelli et al. 2014, and references therein). In the prestellar core phase, the low temperatures and the resulting $\mathrm{CO}$ freeze-out enhance the abundance of the deuterated molecules. These molecules are stored in the icy grain mantles and are then released into the gas phase in the protostellar stage (Ceccarelli et al. 2007; Caselli et al. 2008). This is the case of methanol $\left(\mathrm{CH}_{3} \mathrm{OH}\right)$, which is formed on the grain surfaces (e.g. Tielens 1983; Rimola et al. 2014) and is then released into the gas phase because dust mantles are thermally evaporated in the inner warm regions around low-mass protostars (e.g. Ceccarelli et al. 2000, 2007; Parise et al. 2002, 2004, 2006) and/or sputtered by shocks (Codella et al. 2012; Fontani et al. 2014).

Deuterated methanol has been analysed towards Class 0 protostars (Parise et al. 2006): the measured D/H of 0.4-0.6 indicates that $\mathrm{CH}_{2} \mathrm{DOH}$ can be almost as abundant as $\mathrm{CH}_{3} \mathrm{OH}$. In the only other more evolved source studied so far, the same ratio appears to be lower $\left(\mathrm{D} / \mathrm{H} \sim 1-7 \times 10^{-3}\right)$, suggesting a decrease in deuterated species that is due to evolutionary effects (Bianchi et al. 2017). However, all the existing observations have been performed with single-dish telescopes (angular resolution $\geq 10^{\prime \prime}$ ), which do not distinguish the different components associated with a protostellar system (jet, high-velocity shocks, slower accretion shocks, and inner envelope). Sub-arcsecond resolution 
observations are then crucial to measure the $\mathrm{D} / \mathrm{H}$ ratio within 50-100 au from the protostar, that is, in the region hosting the protoplanetary discs. Dust grains and ices may also be affected by the accretion shock near the centrifugal barrier, therefore we do not know the deuterium fractionation at small scales.

The HH212 star-forming region in Orion $(d=450 \mathrm{pc})$ is an ideal laboratory for studying the jet-disc system. The HH212$\mathrm{mm}$ Class 0 protostar is driving a bright, extended, and bipolar molecular jet extensively observed using IRAM-NOEMA, SMA, and ALMA (e.g. Lee et al. 2006, 2007, 2008, 2015, 2016, 2017a,b; Codella et al. 2007; Cabrit et al. 2007, 2012). A molecular disc was revealed in $\mathrm{HCO}^{+}, \mathrm{C}^{17} \mathrm{O}$, and $\mathrm{SO}$ emission, using the first ALMA cycles with angular resolutions of $\sim 0^{\prime \prime} 6$. Velocity gradients have been detected along the equatorial plane consistent with a rotating disc around a $0.2-0.3 M_{\odot}$ protostar (Lee et al. 2014; Codella et al. 2014; Podio et al. 2015). A disc with a radius of 60 au has indeed been imaged by Lee et al. (2017a), who observed the dust continuum emission at $850 \mu \mathrm{m}$. Finally, Codella et al. (2016) and Leurini et al. (2016) suggested that $\mathrm{HDO}$ and $\mathrm{CH}_{3} \mathrm{OH}$ emission is likely associated with outflowing gas, and possibly with a disc wind. To conclude, the $\mathrm{HH} 212$ region is the unique Class 0 protostellar region where a bright jet, a compact rotating disc, and signatures of a disc wind have been revealed. In this Letter, we exploit ALMA Band 7 observations with an angular resolution down to 0 '! 15 to obtain the first measurement of methanol deuteration in the disc formation region.

\section{Observations}

HH212 was observed in Band 7 with ALMA using $3412 \mathrm{~m}$ antennas between 15 June and 19 July 2014 during the Cycle 1 phase. Further observations of $\mathrm{HH} 212$ were performed in Band 7 with ALMA using $4412 \mathrm{~m}$ antennas between 6 October and 26 November 2016 during the Cycle 4 phase. The maximum baselines for Cycle 1 and 4 were $650 \mathrm{~m}$ and $3 \mathrm{~km}$, respectively.

In Cycle 1 the spectral windows between $337.1-338.9 \mathrm{GHz}$ and $348.4-350.7 \mathrm{GHz}$ were observed using spectral channels of $488 \mathrm{kHz}\left(0.42-0.43 \mathrm{~km} \mathrm{~s}^{-1}\right)$, subsequently smoothed to $1.0 \mathrm{~km} \mathrm{~s}^{-1}$. Calibration was carried out following standard procedures, using quasars J0607-0834, J0541-0541, J0423-013, and Ganymede. The continuum-subtracted images have clean-beam FWHMs from $00^{\prime \prime} .41 \times 0^{\prime \prime} .33$ to $0^{\prime \prime} .52 \times 0^{\prime \prime} .34\left(\mathrm{PA}=-63^{\circ}\right)$, and an rms noise level of $5-6 \mathrm{mJy}_{\text {beam }}{ }^{-1}$ in the $1.0 \mathrm{~km} \mathrm{~s}^{-1}$ channels For Cycle 4 spectral units of $977 \mathrm{kHz}\left(0.87 \mathrm{~km} \mathrm{~s}^{-1}\right)$ were used to observe the spectral windows between $334.1-336.0 \mathrm{GHz}$ Calibration was carried out following standard procedures, using quasars J0510+1800, J0552+0313, J0541-0211, and J05523627. The continuum-subtracted images have a typical cleanbeam FWHM of $0 \prime \prime 15 \times 00^{\prime \prime} 12\left(\mathrm{PA}=-88^{\circ}\right)$ and an rms noise level of $1 \mathrm{mJy}^{\text {beam }}{ }^{-1}$ in the $0.87 \mathrm{~km} \mathrm{~s}^{-1}$ channels. Spectral line imaging was achieved with the CASA package, while the data analysis was performed using the GILDAS ${ }^{1}$ package. Positions are given with respect to the MM1 protostar continuum peak located at $\alpha(\mathrm{J} 2000)=05^{\mathrm{h}} 43^{\mathrm{m}} 51 \mathrm{~s} 41, \delta(\mathrm{J} 2000)=-01^{\circ}$ $02^{\prime}$ 53'.17 (Lee et al. 2014). Lines were identified using spectroscopic parameters extracted from the Jet Propulsor Laboratory (JPL ${ }^{2}$, Pickett et al. 1998) and the Cologne Database for Molecular Spectroscopy (CDMS ${ }^{3}$; Müller et al. 2001, 2005).

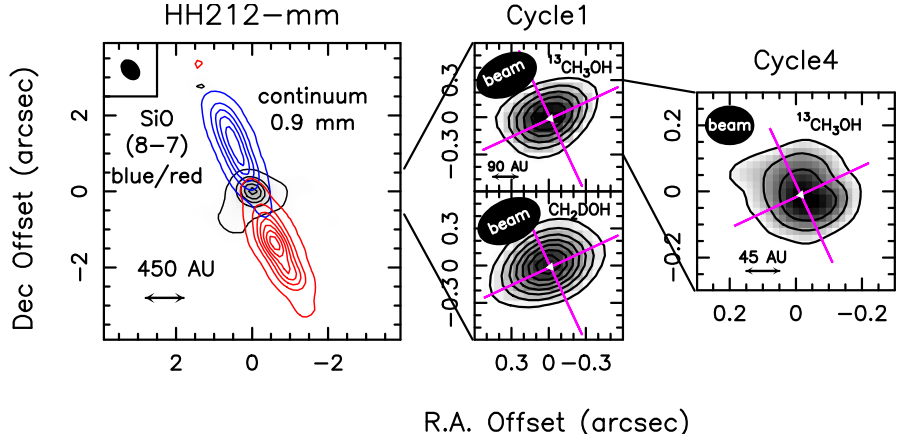

Fig. 1. Left panel: $\mathrm{HH} 212$ protostellar system as observed by ALMABand 7 (Codella et al. 2014). Blue and red contours plot the blue- and redshifted $\mathrm{SiO}(8-7)$ jet overlaid on the continuum at $0.9 \mathrm{~mm}$ (black contours). Positions are with respect to the coordinates reported in Sect. 2. The filled ellipse shows the synthesised beam (HPBW): $00^{\prime \prime} 61 \times 00^{\prime \prime} 45$. Central panels: zoom-in of the central region as observed by ALMABand 7 Cycle 1: ${ }^{13} \mathrm{CH}_{3} \mathrm{OH}\left(13_{0,13}-12_{1,12}\right) \mathrm{E}$ and $\mathrm{CH}_{2} \mathrm{DOH}\left(9_{0,9}-8_{1,8}\right) \mathrm{e} 0$ integrated over $\pm 4 \mathrm{~km} \mathrm{~s}^{-1}$ with respect to the $v_{\text {sys }}=+1.7 \mathrm{~km} \mathrm{~s}^{-1}$, (black contours and grey scale). First contours and steps are $5 \sigma$ for ${ }^{13} \mathrm{CH}_{3} \mathrm{OH}$ $\left(15 \mathrm{mJy}\right.$ beam $\left.{ }^{-1} \mathrm{~km} \mathrm{~s}^{-1}\right)$ and $\mathrm{CH}_{2} \mathrm{DOH}\left(35 \mathrm{mJy}\right.$ beam $\left.{ }^{-1} \mathrm{~km} \mathrm{~s}^{-1}\right)$. The HPBWs are $0^{\prime \prime} .52 \times 00^{\prime \prime} .34\left(\mathrm{PA}=-64^{\circ}\right)$ for ${ }^{13} \mathrm{CH}_{3} \mathrm{OH}$ and $0^{\prime \prime} .54 \times 0^{\prime \prime} .35$ $\left(\mathrm{PA}=-65^{\circ}\right)$ for $\mathrm{CH}_{2} \mathrm{DOH}$. Right panel: zoom-in of the central region, as observed by ALMA-Band 7 Cycle 4, showing the ${ }^{13} \mathrm{CH}_{3} \mathrm{OH}\left(12_{1,11^{-}}\right.$ $12_{0,12}$ )A emission integrated over $10 \mathrm{~km} \mathrm{~s}^{-1}$ around $v_{\text {sys }}$ (black contours and grey scale). First contours and steps are $5 \sigma\left(20 \mathrm{mJy}^{\text {beam }}{ }^{-1} \mathrm{~km} \mathrm{~s}^{-1}\right)$ and $3 \sigma$, respectively. The HPBW is $0^{\prime \prime} .15 \times 0^{\prime \prime} .12\left(\mathrm{PA}=-88^{\circ}\right)$.

\section{Results and discussion}

\subsection{Line spectra and maps}

In Cycle 1 data, we detected 5 lines of ${ }^{13} \mathrm{CH}_{3} \mathrm{OH}$ and 12 lines of $\mathrm{CH}_{2} \mathrm{DOH}$ covering excitation energies, $E_{\text {up }}$, from 17 to $438 \mathrm{~K}$ (see Table A.1). In addition, in the Cycle 4 dataset we revealed one transition of ${ }^{13} \mathrm{CH}_{3} \mathrm{OH}\left(E_{\text {up }}=193 \mathrm{~K}\right)$ and one of $\mathrm{CH}_{2} \mathrm{DOH}\left(E_{\text {up }}=327 \mathrm{~K}\right)$. Examples of the detected line profiles are shown in Fig. A.1: the emission peaks at velocities between $\simeq+1 \mathrm{~km} \mathrm{~s}^{-1}$ and $+3 \mathrm{~km} \mathrm{~s}^{-1}$, consistent with the systemic velocity of $+1.7 \mathrm{~km} \mathrm{~s}^{-1}$ (Lee et al. 2014), when the spectral resolution of $1 \mathrm{~km} \mathrm{~s}^{-1}$ is considered. The profiles, fit using the GILDAS package, are Gaussian with typical full width at halfmaximum (FWHM) $\sim 5 \mathrm{~km} \mathrm{~s}^{-1}$. The spectral parameters of the detected lines are presented in Table A.1. Figure 1 introduces the HH212 system: the $0.9 \mathrm{~mm}$ continuum traces the envelope hosting the protostar. The bipolar blue- and red-shifted lobes are traced by SiO (Codella et al. 2007; Lee et al. 2014). The central panels, covering a zoom-in of the central protostellar region $\left(\lesssim 0^{\prime \prime} 5\right.$, i.e. $225 \mathrm{au}$ ), show the Cycle 1 data, specifically, examples of the emission maps of ${ }^{13} \mathrm{CH}_{3} \mathrm{OH}$ and $\mathrm{CH}_{2} \mathrm{DOH}$. The emission is spatially unresolved with a size smaller than the Cycle 1 beam $\left(0^{\prime \prime} .5 \times 0^{\prime \prime} .3\right)$, corresponding to a size of $225 \times 135$ au. However, the definitely higher angular resolution provided by the Cycle 4 dataset $(0$ '! $15 \times 0$ '! 12 corresponding to a radius of $34 \times 27 \mathrm{au})$ provides spatially resolved images of the ${ }^{13} \mathrm{CH}_{3} \mathrm{OH}$ and $\mathrm{CH}_{2} \mathrm{DOH}{ }^{4}$ emission lines. We used the ${ }^{13} \mathrm{CH}_{3} \mathrm{OH}$ emission as revealed in the Cycle 4 image to show in the right panel of Fig. 1 a further zoom-in, sampling the inner $100 \mathrm{au}$.

\footnotetext{
http://www.iram.fr/IRAMFR/GILDAS

2 https://spec.jpl.nasa.gov/

http://www.astro.uni-koeln.de/cdms/
} 4 The $\mathrm{CH}_{2} \mathrm{DOH}$ line is blended with the $\mathrm{SO}_{2}\left(8_{2,6}-7_{1,7}\right)$ line
(Table A.1). 


\subsection{Methanol deuteration}

We used the ${ }^{13} \mathrm{CH}_{3} \mathrm{OH}$ lines detected in Cycle 1 to perform a rotation diagram analysis, assuming local thermal equilibrium (LTE) conditions and optically thin lines. In order to exclude opacity effects, we used the ${ }^{13} \mathrm{CH}_{3} \mathrm{OH}$ lines to derive the $\mathrm{CH}_{3} \mathrm{OH}$ column density because the main isotopologue lines observed by Leurini et al. (2016) are moderately optically thick $(\tau<0.4)$. We also verified that the $\mathrm{CH}_{2} \mathrm{DOH}$ transitions are indeed optically thin using a population diagram analysis (Goldsmith \& Langer 1999) in which opacities are self-consistently computed. We used ${ }^{13} \mathrm{CH}_{3} \mathrm{OH}$ and $\mathrm{CH}_{2} \mathrm{DOH}$ lines extracted from the same ALMA dataset in order to have the same $u-v$ coverage and to minimise calibration effects. Moreover, the Cycle 0 (beam $\simeq 0^{\prime \prime}$.6) observations of $\mathrm{CH}_{3} \mathrm{OH}$ (Leurini et al. 2016) trace a much warmer component dominated by lines with $E_{\text {up }}$ up to $747 \mathrm{~K}$ with respect to the emission lines analysed in this paper. A source size of 0 '. $19 \pm 0$ 0'.02 has been derived from the ${ }^{13} \mathrm{CH}_{3} \mathrm{OH}$ Cycle 4 map integrated over the whole emission range and then used to correct the Cycle 1 observed values. This value is in good agreement with $\sim 0^{\prime \prime}$. 2 , as derived from $\mathrm{CH}_{3} \mathrm{OH}$ (Leurini et al. 2016). Figure A.2 shows the derived rotational temperature and column density, which are $T_{\text {rot }}=(171 \pm 52) \mathrm{K}$ and $N_{\text {tot }}=(6.5 \pm 2.1) \times 10^{16} \mathrm{~cm}^{-2}$, respectively. Conservatively, we used only Cycle 1 data to perform the fit; however, the ${ }^{13} \mathrm{CH}_{3} \mathrm{OH}$ Cycle 4 line is in good agreement with Cycle 1 observations. In the lower panel of Fig. A.2 we derive the column density of $\mathrm{CH}_{2} \mathrm{DOH}$, assuming the same rotational temperature and size derived for ${ }^{13} \mathrm{CH}_{3} \mathrm{OH}$. We excluded from the fitting the two $\mathrm{CH}_{2} \mathrm{DOH}$ transitions with $E_{\text {up }}>300 \mathrm{~K}$ to exclude any possible contamination from non-thermal excitation processes or the occurrence of a component with a different higher excitation condition. However, the fitting obtained with the low-energy transitions (continuous line) is in agreement with the two excluded transitions, as well as with the Cycle 4 transition, as shown by the dotted line. The derived column density for $\mathrm{CH}_{2} \mathrm{DOH}$ is then $N_{\text {tot }}=(1.1 \pm 0.2) \times 10^{17} \mathrm{~cm}^{-2}$. We note that an LVG analysis (see Ceccarelli et al. 2003, for further details) of the ${ }^{13} \mathrm{CH}_{3} \mathrm{OH}$ emission indicates a source size of $0^{\prime \prime} .1-0^{\prime \prime} .2, T_{\text {kin }}$ larger than $100 \mathrm{~K}$ and densities higher than $10^{6} \mathrm{~cm}^{-3}$, in agreement with the HDO results reported by Codella et al. (2016).

The column densities derived from the rotation diagrams were used to derive the methanol $\mathrm{D} / \mathrm{H}$ for the first time in the inner 100 au around a low-mass protostar. When we assume a ${ }^{12} \mathrm{C} /{ }^{13} \mathrm{C}$ ratio of 70 (Milam et al. 2005) at the galactocentric distance of $\mathrm{HH} 212(\sim 8.3 \mathrm{kpc})$, the $\mathrm{D} / \mathrm{H}$ is $(2.4 \pm 0.4) \times 10^{-2}$. This value is in agreement with the upper limit of 0.27 derived by Lee et al. (2017b) using $\mathrm{CH}_{2} \mathrm{DOH}$ and three lines of $\mathrm{CH}_{3} \mathrm{OH}$. In addition, our measurement is lower than previous values of $\mathrm{CH}_{2} \mathrm{DOH}$ in other Class 0 objects performed with the IRAM $30 \mathrm{~m}$ single dish, which indicate $\mathrm{D} / \mathrm{H} \simeq 40-60 \%$ (Parise et al. 2006). Taking into account that as reported by Belloche et al (2016), the column densities of $\mathrm{CH}_{2} \mathrm{DOH}$ derived in Parise et al. (2006) were overestimated by a factor $\sim 2$ because of a problem in the spectroscopic parameters, the $\mathrm{HH} 212$ deuteration is still one order of magnitude lower. What are the reasons of this difference? To start with, the single-dish measurements by Parise et al. (2006) are sampling regions $\geq 2000$ au: the $\mathrm{D} / \mathrm{H}$ is calculated assuming that the main isotopologue and the deuterated species come from the same emitting source, but this cannot be verified with low angular resolutions. This issue is overcome with high angular resolution observations, like those presented here, which allow us to directly image the emitting region. Interestingly, Jørgensen et al. (2016) reported a level of deuteration for glycolaldehyde of 5\% in IRAS 16293 in Ophiuchus on a scale of $50 \mathrm{au}$. That said, a possible explanation for the lower methanol deuteration measured for $\mathrm{HH} 212 \mathrm{~mm}$ could be related to different physical conditions during the formation of methanol on dust mantles during the prestellar phase. A higher gas temperature reduces the atomic gas $\mathrm{D} / \mathrm{H}$ ratio landing on the grain surfaces and in turn reduces the deuteration of methanol. All the sources observed by Parise et al. (2006) are located in the Perseus star-forming region, which could have had experienced different conditions with respect to the Orion B region where HH212 lies. Specifically, while in Perseus the dust temperature is about $\sim 12 \mathrm{~K}$ (Zari et al. 2016), HH212 is located about one degree north of the high-mass star-forming region NGC 2024, and the dust temperature here is $\geq 16 \mathrm{~K}$ (Lombardi et al. 2014). As shown by Taquet et al. (2012, 2014), for instance, after the volume density is fixed, deuteration (including that of methanol) decreases as temperature increases. The models indicate that $\mathrm{D} / \mathrm{H}$ can decrease by up to one order of magnitude by increasing the temperature from $10 \mathrm{~K}$ to $20 \mathrm{~K}$. Interestingly, we note that according to Fuente et al. (2014), the methanol deuteration in the hot core in the intermediate-mass star-forming region NGC 7129, FIRS 2 is $2 \%$. However, in hot cores associated with massive star-forming regions in Orion (Peng et al. 2012), the methanol $\mathrm{D} / \mathrm{H}$ is between $0.8 \times 10^{-3}$ and $1.3 \times 10^{-3}$, which is lower by one order of magnitude than the values reported here.

\subsection{Emitting region of deuterated methanol}

Figure 2 shows the channel maps of the two transitions observed during ALMA Cycle 4, that is, at the highest spatial resolution presented here: ${ }^{13} \mathrm{CH}_{3} \mathrm{OH}\left(12_{1,11}-12_{0,12}\right) \mathrm{A}$ and $\mathrm{CH}_{2} \mathrm{DOH}$ $\left(16_{2,15}-16_{1,15}\right)$ o1 (see Table A.1). For both lines the rightmost panels show the spatial distributions of the emission close to the systemic velocity. The remaining panels are for the blue- and redshifted velocities, imaged up to $\pm 4 \mathrm{~km} \mathrm{~s}^{-1}$. We note that for $\mathrm{CH}_{2} \mathrm{DOH}$ the redshifted emission is partially contaminated by the $\mathrm{SO}_{2}\left(8_{2,6}-7_{1,7}\right)$ emission (see Table A.1 and Fig. A.1).

The spatial distribution of the methanol isotopologues at the systemic velocity is elongated along the jet axis $(\mathrm{PA}=$ $\left.22^{\circ}\right)$. A fit in the image plane gives for ${ }^{13} \mathrm{CH}_{3} \mathrm{OH}$ a beamdeconvolved FWHM size of $0{ }^{\prime \prime} 18\left(00^{\prime \prime} 02\right) \times 00^{\prime \prime} 12\left(0^{\prime \prime}, 02\right)$ at $\mathrm{PA}=$ $33^{\circ}$. For $\mathrm{CH}_{2} \mathrm{DOH}$ we derived $0^{\prime \prime} \cdot 20\left(0^{\prime \prime}, 02\right) \times 00^{\prime \prime} \cdot 10\left(0^{\prime \prime} .02\right)$ and $\mathrm{PA}=19^{\circ}$. These values correspond to FWHM sizes of $81 \times 54$ au $\left({ }^{13} \mathrm{CH}_{3} \mathrm{OH}\right)$ and $90 \times 45$ au $\left(\mathrm{CH}_{2} \mathrm{DOH}\right)$. Closer to the blue- and redshifted emission, we resolve a clear velocity gradient parallel to the equatorial plane (see also Fig. A.3) with shifts of \pm 0 '” $1=$ $45 \mathrm{au}$, in the same sense as the HH212 disc rotation. The typical (beam-deconvolved) size of the blue- and redshifted emission at $\pm 2 \mathrm{~km} \mathrm{~s}^{-1}$ from systemic is 0 '.22(0,.04) $\times 0$ ". $14\left(0^{\prime \prime} .04\right)$ and $0^{\prime \prime} .18\left(0^{\prime \prime} .02\right) \times 00^{\prime \prime} .10\left(0^{\prime \prime} .03\right)$, respectively, similar to the size at $V_{\text {sys }}$. Rotation has previously been noted in $\mathrm{CH}_{3} \mathrm{OH}$ by Leurini et al. (2016) from unresolved maps at lower resolution $\left(\simeq 00^{\prime \prime} 6\right)$. The authors further noted that emission centroids (fitted in $u-v$ plane) moved away from the source, and eventually away from the mid plane, at higher velocity, speculating that the methanol emission is not dominated by a Keplerian disc or the rotatinginfalling cavity, and possibly associated with a disc wind. With the present images we reach higher angular resolution, which allows us to refine the picture: the systemic velocity appears to arise mainly from two peaks at $\pm 0,05=20$ au above and below the disc plane (of which the centroids in Leurini et al. 2016 only traced the barycentre). In addition, channel maps at $\pm 1 \mathrm{~km} \mathrm{~s}^{-1}$ suggest redshifted emission peaking in the north and blueshifted emission peaking in the south, which is not the sense 


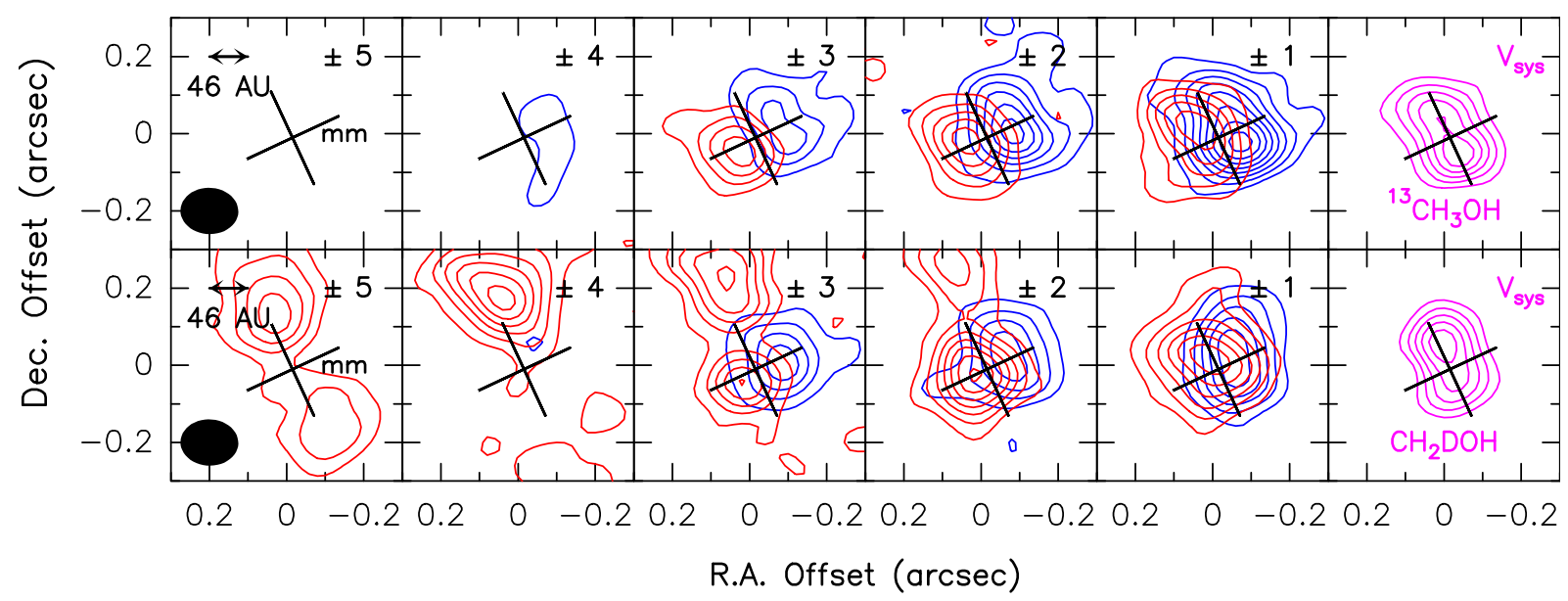

Fig. 2. Channel maps of the ${ }^{13} \mathrm{CH}_{3} \mathrm{OH}\left(12_{1,11}-12_{0,12}\right) \mathrm{A}$ (upper panels) and $\mathrm{CH}_{2} \mathrm{DOH}\left(16_{2,15}-16_{1,15}\right)$ o1 (lower panels) blue- and redshifted (continuum subtracted) emissions observed during ALMA-Cycle 4 towards the HH212 mm protostar. Each panel shows the emission integrated over a velocity interval of $1 \mathrm{~km} \mathrm{~s}^{-1}$ shifted with respect to the systemic velocity (see the magenta channel, sampling the velocity between $+1.5 \mathrm{~km} \mathrm{~s}^{-1}$ and $+2.5 \mathrm{~km} \mathrm{~s}^{-1}$ ) by the value given in the upper-right corner. The black cross (inclined in order to point the SiO jet direction and consequently the equatorial plane, see Fig. 1) indicates the position of the protostar. The ellipse in the top left panel shows the ALMA synthesised beam (HPBW): $0^{\prime \prime} .15 \times 00^{\prime \prime} 12\left(\mathrm{PA}=-88^{\circ}\right)$. First contours and steps correspond to $3 \sigma\left(2.7 \mathrm{mJy}\right.$ beam $\left.{ }^{-1} \mathrm{~km} \mathrm{~s}^{-1}\right)$. Note that for $\mathrm{CH}_{2} \mathrm{DOH}$ at velocities higher than $+1.0 \mathrm{~km} \mathrm{~s}^{-1}$ a further emission appears along the jet axes to the north and to the south with respect to the $\mathrm{CH}_{2} \mathrm{DOH}$ emission. This is due to the $\mathrm{SO}_{2}\left(8_{2,6}-7_{1,7}\right)$ emission line (see Table 1 and Fig. 2), which has been confirmed to be a good tracer of outflowing material (Podio et al. 2015). Given that the velocity scale has been derived by first fixing the $\mathrm{CH}_{2} \mathrm{DOH}$ frequency, the $\mathrm{SO}_{2}$ emission looks artificially red, but it is clearly blueshifted (see Fig. A.1).

we would naively expect for a wind from HH212 mm. Since the jet axis is so close to the plane of the $\mathrm{sky}^{5}$, this might perhaps still be explained by non-axisymmetric structure in a disc wind, but synthetic predictions would be needed to test this. Alternatively, our data suggest that the emission could arise from accretion shocks occurring at the centrifugal barrier associated with the accretion disc, and heating the gas at temperatures around $100 \mathrm{~K}$ (Sakai et al. 2014; Oya et al. 2016). The radial extent $\sim 50$ au of the observed red- and blueshifted emission is indeed consistent with the dust disc radius $\sim 60$ au, recently resolved by Lee et al. (2017a) at $850 \mu \mathrm{m}$ with ALMA. In this case, we would observe a thicker accretion shock near the centrifugal barrier in $\mathrm{HH} 212$, as recently seen in $\mathrm{C}_{2} \mathrm{H}$ in L1527 (Sakai et al. 2017). We may also have a contribution from a warm disc atmosphere, emitting in the northern/southern portions of the accretion disc, thicker than the dust atmosphere.

\section{Conclusions}

The ALMA Cycle 1 and 4 observations allow us to measure methanol deuteration in the inner 50 au of the jet-disc system associated with the Class 0 protostar $\mathrm{HH} 212$, in Orion B. The deuteration is $\simeq 2 \times 10^{-2}$, a value lower than has previously been measured using single-dish telescopes towards Class 0 protostars in Perseus. Although we cannot exclude that single-dish observations are mixing different gas components with different $\mathrm{D} / \mathrm{H}$ values, our findings are consistent with a higher dust temperature in Orion B than in the Perseus cloud. This confirms the diagnostic value of molecular deuteration for recovering the physical conditions during the pre-collapse phase. The emission is confined in a rotating structure that extends at \pm 45 au from the equatorial plane and is elongated along the jet axis. Disc wind, disc atmosphere, and accretion shocks could explain the observed images. Higher spatial resolution maps are necessary to distinguish between these possibilities.

5 The system inclination is $4^{\circ}$ to the plane of sky (Claussen et al. 1998).
Acknowledgements. We thank C.-F. Lee for instructive comments and suggestions. We also thank the anonymous referee for having improved the manuscript. This paper makes use of the ADS/JAO.ALMA\#2012.1.00997.S and ADS/JAO.ALMA\#2016.1.01475.S data (PI: C. Codella). ALMA is a partnership of ESO (representing its member states), NSF (USA) and NINS (Japan), together with NRC (Canada) and NSC and ASIAA (Taiwan), in cooperation with the Republic of Chile. The Joint ALMA Observatory is operated by ESO, AUI/NRAO and NAOJ. This work was supported (i) by the Italian Ministero dell'Istruzione, Università e Ricerca (MIUR) through the grant Progetti Premiali 2012 - iALMA (CUP C52I13000140001); and (ii) by the program PRIN-MIUR 2015 STARS in the CAOS (Simulation Tools for Astrochemical Reactivity and Spectroscopy in the Cyberinfrastructure for Astrochemical Organic Species, 2015F59J3R, MIUR e della Scuola Normale Superiore). M.T. aknowledges partial support from the project AYA2016-79006-P.

\section{References}

Belloche, A., Müller, H. S. P., Garrod, R. T., \& Menten, K. M. 2016, A\&A, 587, A91

Bianchi, E., Codella, C., Ceccarelli, C., et al. 2017, MNRAS, 467, 3011

Cabrit, S., Codella, C., Gueth, F., et al. 2007, A\&A, 468, L29

Cabrit, S., Codella, C., Gueth, F., \& Gusdorf, A. 2012, A\&A, 548, L2

Caselli, P., Vastel, C., Ceccarelli, C., et al. 2008, A\&A, 492, 703

Ceccarelli, C., Loinard, L., Castets, A., et al., 2000, A\&A, 357, L9

Ceccarelli, C., Maret, S., Tielens, A. G. G. M., Castets, A., \& Caux, E. 2003, A\&A, 410, 587

Ceccarelli, C., Caselli, P., Herbst, E., Tielens, A. G. G. M., \& Caux, E. 2007, Protostars and Planets V, eds. H. Beuther, R. Klessen, C. Dullemond, \& Th. Henning (University of Arizona Press), 47

Ceccarelli, C., Caselli, P., Bockelee-Morvan, D. et al. 2014, in Protostars and Planets VI, eds. H. Beuther, R. Klessen, C. Dullemond, \& Th. Henning (University of Arizona Press), 859

Claussen, M. J., Marvel, K. B., Wootten, A., \& Wilking, B. A. 1998, ApJ, 507, L79

Codella, C., Cabrit, S., Gueth, F., et al. 2007, A\&A, 462, L53

Codella, C., Ceccarelli, C., Lefloch, B., et al. 2012, ApJ, 757, L9

Codella, C., Cabrit, S., Gueth, F., et al. 2014, A\&A, 568, L5

Codella, C., Ceccarelli, C., Cabrit, S., et al. 2016, A\&A, 586, L3

Fontani, F., Codella, C., Lefloch, B., et al. 2014, ApJ, 788, L43

Fuente, A., Cernicharo, J., Caselli, P., et al. 2014, A\&A, 568, A65

Goldsmith, P. F., \& Langer, W. D. 1999, ApJ, 517, 209

Jørgensen, J., van der Wiel, M. H. D., Coutens, A., et al. 2016, A\&A, 595, 117

Lee, C.-F., Ho, P. T. P., Beuther, H., et al. 2006, ApJ, 639, L292 
E. Bianchi et al.: Deuterated methanol on a solar system scale around the HH212 protostar

Lee, C.-F., Ho, P. T. P., Hirano, N., et al. 2007, ApJ, 659, L499

Lee, C.-F., Ho, P. T. P., Bourke, T. L., et al. 2008, ApJ, 685, 1026

Lee, C.-F., Hirano, N., Zhang, Q., et al. 2014, ApJ, 786, 114

Lee, C.-F., Hirano, N., Zhang, Q., et al. 2015, ApJ, 805, 186

Lee, C.-F., Li, Z.-Y., Ho, P.-T.-P., et al. 2017a, Nature, 1, 0152

Lee, C.-F., Li, Z.-Y., Ho, P.-T.-P., et al. 2017b, ApJ, 843, L27

Leurini, S., Codella, C., Cabrit, S., et al. 2016, A\&A, 595, L4

Lombardi, M., Bouy, H., Alves, J., \& Lada, C. J. 2014, A\&A, 566, A45

Milam, S. N., Savage, C., Brewster, M. A., Ziurys, L. M., \& Wyckoff, S., 2005, ApJ, 634, 1126

Müller, H. S. P., Thorwirth, S., Roth, D. A., et al. 2001, A\&A, 370, L49

Müller, H. S. P., Schlöder, F., Stutzki, J., et al. 2005, J. Mol. Struct., 742, 215

Oya, Y., Sakai, N., López-Sepulcre, A., et al. 2016, ApJ, 824, 88

Parise, B., Ceccarelli, C., Tielens, A. G. G. M., et al. 2002, A\&A, 393, L49
Parise, B., Castets, A., Herbst, E., et al. 2004, A\&A, 416, 159

Parise, B., Ceccarelli, C., Tielens, A. G. G. M., et al. 2006, A\&A, 453, 949

Peng, T.-C., Despois, D., Brouillet, N., Parise, B., \& Baudry, A. 2012, A\&A, 543, A 152

Pickett, H. M., Poynter, R. L., Cohen, E. A., et al. 1998, J. Quant. Spectr. Rad. Transf., 60, 883

Podio, L., Codella, C., Gueth, F., et al. 2015, A\&A, 581, A85

Rimola, A., Taquet, V., Ugliengo, P, et al., 2014, A\&A, 572, A70

Sakai, N., Sakai, T., Hirota, T., et al. 2014, Nature, 507, 78

Sakai, N., Oya, Y., Higuchi, E. A., et al. 2017, MNRAS, 467, L76

Taquet, V., Ceccarelli, C., \& Kahane, C. 2012, ApJ, 784, L3

Taquet, V., Charnley, S. B., Sipilä, O. 2014, ApJ, 791, 1

Tielens, A. G. G. M. 1983, A\&A, 119, 177

Zari, E., Lombardi, M., Alves, J., Lada, C. J., \& Bouy, H. 2016, A\&A, 587, A106 


\section{Appendix A: Additional material}

Table A.1 lists all the methanol isotopologue lines observed towards HH212 mm during ALMA Cycle 1 and Cycle 4 operations. Figure A.1 shows examples of line profiles in $T_{B}$ scale, while Fig. A.2 shows the rotation diagrams for ${ }^{13} \mathrm{CH}_{3} \mathrm{OH}$ and $\mathrm{CH}_{2}$ DOH. Figure A.3 shows the ${ }^{13} \mathrm{CH}_{3} \mathrm{OH}\left(12_{1,11}-12_{0,12}\right) \mathrm{A}$ spectrum extracted at \pm 0 '.06 from the protostar, in the equatorial plane direction.

Table A.1. Emission lines detected in ALMA Cycle 1 and 4 measurements towards $\mathrm{HH} 212 \mathrm{~mm}$.

\begin{tabular}{lccccc}
\hline \hline \multicolumn{1}{c}{ Transition } & $\begin{array}{c}v^{a} \\
(\mathrm{GHz})\end{array}$ & $\begin{array}{c}E_{\text {up }}{ }^{a} \\
(\mathrm{~K})\end{array}$ & $\begin{array}{c}S \mu^{2 a} \\
\left(\mathrm{D}^{2}\right)\end{array}$ & $\begin{array}{c}\mathrm{rms} \\
(\mathrm{K})\end{array}$ & $\begin{array}{c}F_{\text {int }}{ }^{b} \\
\left(\mathrm{~K} \mathrm{~km} \mathrm{~s}^{-1}\right)\end{array}$ \\
\hline${ }^{13} \mathrm{CH}_{3} \mathrm{OH} \mathrm{Cycle} 1$ \\
\hline $13_{0,13}-12_{1,12} \mathrm{~A}$ & 338.75995 & 206 & 13 & 0.29 & $9.6(1.1)$ \\
$13_{4,9}-14_{3,11} \mathrm{E}$ & 347.78840 & 303 & 4 & 0.36 & $4.0(1.4)$ \\
$11_{0,11}-10_{1,9} \mathrm{E}$ & 348.10019 & 162 & 5 & 0.84 & $7.5(3.0)$ \\
$1_{1,1}-0_{0,0} \mathrm{~A}$ & 350.10312 & 17 & 2 & 1.16 & $7.4(3.0)$ \\
$8_{1,7}-7_{2,5} \mathrm{E}$ & 350.42158 & 103 & 2 & 0.93 & $8.7(2.6)$ \\
\hline \multicolumn{7}{c}{$\mathrm{CH}_{2} \mathrm{DOH} \mathrm{Cycle} 1$} \\
$9_{0,9}-8_{1,8} \mathrm{e} 0$ & 337.34866 & 96 & 6 & 0.15 & $15.3(0.5)$ \\
$9_{1,8}-8_{2,6} \mathrm{O} 1^{c}$ & 338.46254 & 120 & 2 & 0.26 & $10.7(2.1)^{c}$ \\
$13_{1,12}-12_{0,12} \mathrm{e} 0$ & 338.86898 & 202 & 2 & 0.13 & $9.7(0.4)$ \\
$6_{1,6}-5_{0,5} \mathrm{e} 0$ & 338.95711 & 48 & 5 & 0.29 & $16.7(0.9)$ \\
$18_{4,15}-18_{3,15} \mathrm{e} 1$ & 347.76728 & 438 & 10 & 0.09 & $6.6(0.3)$ \\
$18_{4,14}-18_{3,16} \mathrm{e} 1$ & 347.95281 & 438 & 10 & 0.18 & $6.4(0.6)$ \\
$4_{1,3}-4_{0,4} \mathrm{e} 1$ & 348.16076 & 38 & 4 & 0.15 & $18.1(0.5)$ \\
$7_{4,4}-7_{3,4} \mathrm{e} 1$ & 349.95168 & 132 & 3 & 0.16 & $25.3(0.5)$ \\
$7_{4,3}-7_{3,5} \mathrm{e} 1$ & 349.95272 & 132 & 3 & & \\
$6_{4,3}-6_{3,3} \mathrm{e} 1$ & 350.02735 & 117 & 2 & 0.23 & $19.5(0.7)$ \\
$6_{4,2}-6_{3,4} \mathrm{e} 1$ & 350.02777 & 117 & 2 & & \\
$5_{4,2}-5_{3,2} \mathrm{e} 1$ & 350.09024 & 104 & 2 & 0.63 & $20.0(2.2)$ \\
$5_{4,1}-5_{3,3} \mathrm{e} 1$ & 350.09038 & 104 & 2 & & \\
$6_{2,5}-5_{1,5} \mathrm{e} 1^{c}$ & 350.45387 & 72 & 4 & 0.63 & $17.0(3.9)^{c}$ \\
$5_{1,4}-5_{0,5} \mathrm{e} 1$ & 350.63207 & 49 & 5 & 0.60 & $21.0(2.0)$ \\
\hline $12_{1,11}-12_{0,12} \mathrm{~A}$ & 335.56021 & 193 & 23 & 0.81 & $61.7(2.7)$ \\
\hline $16_{2,15}-16_{1,15}$ o1 ${ }^{d}$ & 334.68395 & 327 & 8 & 0.67 & $51.1(2.9)^{d}$ \\
\hline & \multicolumn{6}{c}{$\mathrm{CH}_{2} \mathrm{DOH} \mathrm{Cycle} 4$} & & \\
\hline
\end{tabular}

Notes. ${ }^{(a)}$ Frequencies and spectroscopic parameters are extracted from the Jet Propulsion Laboratory molecular database (JPL, Pickett et al. 1998) and from the Cologne Database for Molecular Spectroscopy (CDMS, Müller et al. 2001). Upper level energies refer to the ground state of each symmetry. ${ }^{(b)}$ Gaussian fit. ${ }^{(c)}$ The line transition $\left(9_{1,8^{-}}\right.$ $\left.8_{2,6}\right) \mathrm{o} 1$ is partially blended with the $\left(7_{-5,2}-6_{-5,1}\right) \mathrm{E} 1 \mathrm{CH}_{3} \mathrm{OH}$ transition. The line $\left(6_{2,5}-5_{1,5}\right) \mathrm{e} 1$ is partially blended with the $\left(18_{1,17}-17_{1,16}\right) \mathrm{E}$ $\mathrm{CH}_{3} \mathrm{CHO}$ transition. ${ }^{(d)}$ The $\mathrm{CH}_{2} \mathrm{DOH}\left(16_{2,15}-16_{1,15}\right)$ o1 line $\left(E_{\mathrm{u}}=\right.$ $327 \mathrm{~K}, S_{i j} \mu^{2}=8 D^{2}$ ) is partially blended with the $\mathrm{SO}_{2}(8-7)$ transition.
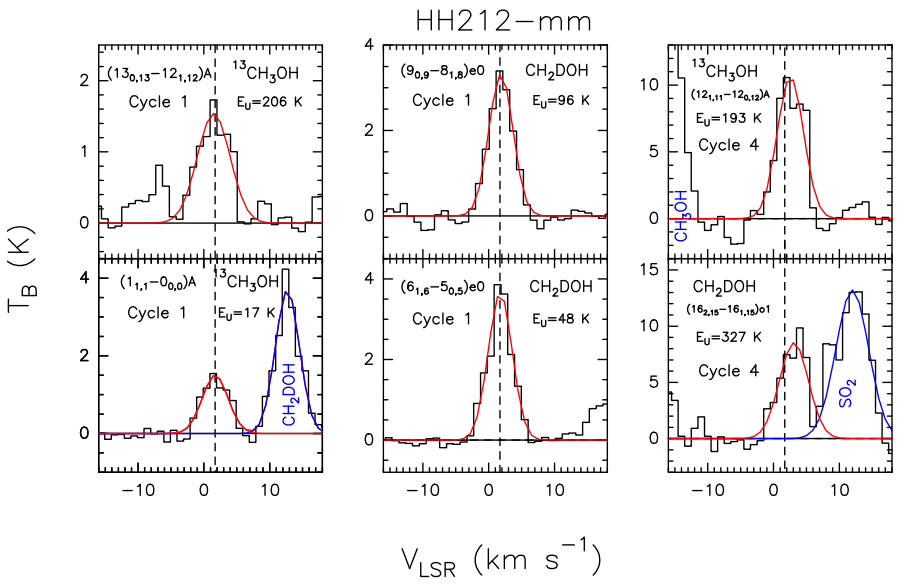

Fig. A.1. Examples of line profiles in $T_{B}$ scale (not corrected for the beam dilution): species and transitions are reported. The vertical dashed line stands for the systemic $V_{\mathrm{LSR}}$ velocity $\left(+1.7 \mathrm{~km} \mathrm{~s}^{-1}\right.$, Lee et al. 2014). Red and blue curves are for the Gaussian fit. In the spectrum of the Cycle $1\left(1_{1,1}-0_{0,0}\right) \mathrm{A}{ }^{13} \mathrm{CH}_{3} \mathrm{OH}$ transition, a $\mathrm{CH}_{2} \mathrm{DOH}$ doublet containing the $\left(5_{4,2}-5_{3,2}\right) \mathrm{e} 1$ and the $\left(5_{4,1}-5_{3,3}\right) \mathrm{e} 1$ transitions is also shown (see Table A.1). In Cycle 4 spectra the $\left(7_{1,7}-6_{1,6}\right) \mathrm{A} \mathrm{CH}_{3} \mathrm{OH}$ and the $\left(8_{2,6^{-}}\right.$ $\left.7_{1,7}\right) \mathrm{SO}_{2}$ transitions are also shown.
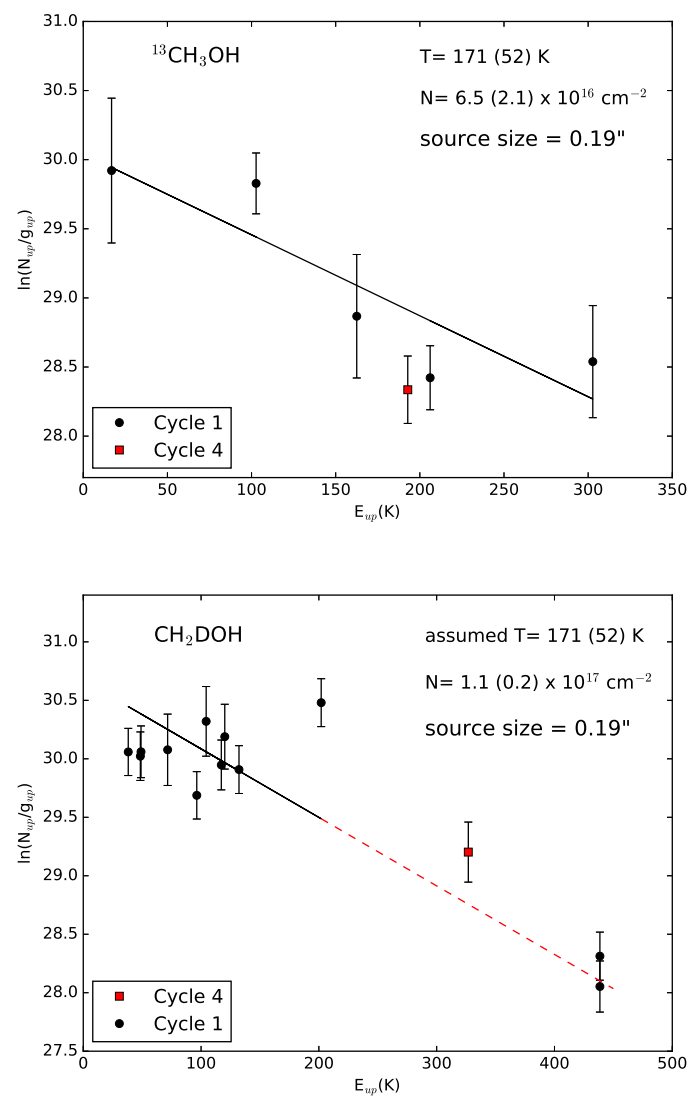

Fig. A.2. Rotation diagrams for ${ }^{13} \mathrm{CH}_{3} \mathrm{OH}$ (upper panel) and $\mathrm{CH}_{2} \mathrm{DOH}$ (lower panel). An emitting region size of $00^{\prime \prime} 19$ is assumed (see text). The parameters $N_{\mathrm{u}}, g_{\mathrm{u}}$, and $E_{\mathrm{up}}$ are the column density, the degeneracy, and the energy of the upper level, respectively. The derived value of the rotational temperature is reported for ${ }^{13} \mathrm{CH}_{3} \mathrm{OH}$. For $\mathrm{CH}_{2} \mathrm{DOH}$ the $T_{\text {rot }}$ derived from the ${ }^{13} \mathrm{C}$-isotopologues is assumed to derive the column density. The two $\mathrm{CH}_{2} \mathrm{DOH}$ transitions with excitation energies higher than $400 \mathrm{~K}$ as well as the Cycle 4 point are excluded from the fit, although they are in good agreement with it. 
E. Bianchi et al.: Deuterated methanol on a solar system scale around the HH212 protostar

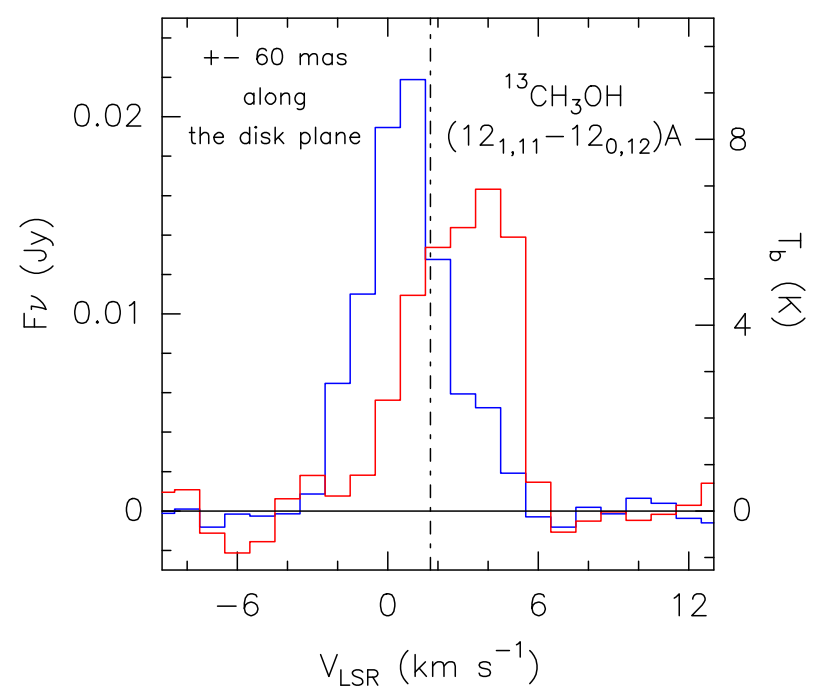

Fig. A.3. Comparison in flux density and in $T_{\mathrm{B}}$ scales $\left(T_{\mathrm{B}} / F_{y}=\right.$ $\left.482.609 \mathrm{~K} \mathrm{Jy}^{-1}\right)$ between the ${ }^{13} \mathrm{CH}_{3} \mathrm{OH}\left(12_{1,11}-12_{0,12}\right) \mathrm{A}$ spectrum extracted at \pm 0 '”06 from the protostar (see Fig. 2: blue- and redshifted emission towards north-west and south-east, respectively) in the direction along the equatorial plane (i.e. disc plane; Lee et al. 2017). The vertical dashed line stands for the systemic $V_{\mathrm{LSR}}$ velocity $\left(+1.7 \mathrm{~km} \mathrm{~s}^{-1}\right.$, Lee et al. 2014). 